\title{
Costo-utilità di dalbavancina versus standard of care (SoC) in pazienti con ABSSSI non severa in Italia
}

\author{
Andrea Marcellusi ${ }^{1,2}$, Chiara Bini ${ }^{1}$, Maria A Rotundo ${ }^{1}$, Rosario Cultrera ${ }^{3}$, Francesco S Mennini ${ }^{1,2}$ \\ ${ }^{1}$ Faculty of Economics, Economic Evaluation and HTA (EEHTA), CEIS, University of Rome "Tor Vergata", Rome - Italia \\ ${ }^{2}$ Institute for Leadership and Management in Health - Kingston University London, London - UK \\ ${ }^{3}$ Dipartimento di Morfologia, Chirurgia e Medicina sperimentale, Università degli Studi di Ferrara, Ferrara - Italia
}

\begin{abstract}
Cost-utility of dalbavancin in patients with non-severe ABSSSI in Italy
Introduction: Acute Bacterial Skin and Skin Structure Infections (ABSSSIs) include all complicated skin and soft tissue infections. The aim of this study was to conduct a cost-utility analysis to compare dalbavancin with standard antibiotic therapies for the management of non-severe ABSSSIs from the National Health Service (NHS) perspective. Methods: A probabilistic decision tree model was developed considering a 30-days follow-up to simulate the therapeutic pathway of a patient treated with dalbavancin or Standard of Care (SoC). The model considered three mutually exclusive health states: a) discharge of patients from the emergency department, b) discharge of patients after one night from admission, c) discharge after 24 or 36 hours from admission. A one-way deterministic sensitivity analysis and a probabilistic sensitivity analysis were conducted.

Results: The analysis showed that the use of dalbavancin in patients with non-severe ABSSSI compared to SoC could generate a reduction in costs $(-€ 291.6$ per patient treated) and an increase in QALYs (+0.0018 per patient treated). In $99.7 \%$ of the simulations carried out, dalbavancin was dominant compared to the SoC. Considering a threshold for the willingness to pay of $€ 30,000$ for QALY gained, the minimum level of efficacy of dalbavancin so that the treatment can be considered cost-effective compared to the SoC was equal to $69.4 \%$.

Conclusions: The analysis showed that dalbavancin may represent a cost-effective option compared to SoC for the treatment of patients with non-severe ABSSSI.
\end{abstract}

Keywords: Cost-effectiveness analysis, Cost-utility analysis, Dalbavancin, Staphylococcal skin diseases

\section{Introduzione}

Le Acute Bacterial Skin and Skin Structure Infections (ABSSSI) comprendono tutte le infezioni della cute e dei tessuti molli complicate. Questo termine è stato introdotto nel 2013 dalla Food and Drug Administration (FDA), che ha redatto nuove linee guida sulla classificazione di quelle che fino a quel momento erano note come Infezioni Batteriche della Cute e dei Tessuti Molli (SSTI, Skin and Soft Tissue Infections) (1). All'interno della nuova categoria delle ABSSSI sono state inserite tutte quelle infezioni che con la precedente classificazione venivano indicate come uncomplicated Skin

Received: April 20, 2020

Accepted: October 5, 2020

Published online: November 6, 2020

Corresponding author:

Andrea Marcellusi

Economic Evaluation and HTA (EEHTA) - Faculty of Economics

Università "Tor Vergata"

Via Columbia 2

00133 Roma - Italia

andrea.marcellusi@uniroma2.it and Skin Structure Infections (uSSSI) e complicated Skin and Skin Structure Infections (cSSSI).

Le infezioni non complicate identificavano patologie cutanee di minore gravità come ascessi minori, impetigine, foruncoli e celluliti, mentre tra quelle complicate venivano inserite le infezioni più serie come ulcere, ustioni e ascessi maggiori, che in generale necessitavano di un ricovero e di un intervento chirurgico.

La nuova classificazione introdotta dalla FDA comprende tutte le infezioni con una superficie minima di $75 \mathrm{~cm}^{2}$. Questa dimensione è stata determinata per distinguere gli ascessi cutanei minori (con una superficie inferiore a $75 \mathrm{~cm}^{2}$ ) dagli ascessi cutanei maggiori (con una superficie superiore a $75 \mathrm{~cm}^{2}$ ).

Dunque, secondo la nuova terminologia introdotta dalla FDA (1), le ABSSSI possono essere suddivise in:

- cellulite/erisipela: infezione cutanea diffusa con aree di arrossamento, edema o indurimento;

- ferita infetta: infezione caratterizzata da una secrezione purulenta generata da una ferita che presenta arrossamento, edema o indurimento;

- ascesso cutaneo maggiore: infezione caratterizzata da una raccolta di pus all'interno del derma o più in profondità, accompagnata da rossore, edema o indurimento. 
Generalmente la causa delle ABSSSI può essere individuata in alcuni patogeni che sono i principali responsabili di queste patologie; in particolare si fa riferimento a patogeni Gram-positivi, come lo Staphylococcus aureus (S. aureus) e lo Streptococcus pyogenes, e meno frequentemente a patogeni Gram-negativi e a batteri anaerobi (spesso in un contesto polimicrobico).

Nella maggioranza dei casi il responsabile principale è lo S. aureus (coinvolto in circa il $40 \%$ dei casi e primo agente patogeno di infezioni cutanee e dei tessuti molli in Nord America, America Latina ed Europa) contro il quale nel corso della storia si sono susseguite varie tipologie di antibiotico quali ad esempio la penicillina e, successivamente, la meticillina.

Secondo le recenti linee guida per la diagnosi e la gestione delle infezioni della cute e dei tessuti molli della Infectious Diseases Society of America (2), le ABSSSI sono classificate sulla base della gravità clinica in infezioni lievi, moderate o severe. Le ABSSSI severe sono quelle che non rispondono al trattamento antibiotico empirico e/o al drenaggio chirurgico e sono generalmente associate a segni di infezione sistemica (febbre $>38^{\circ} \mathrm{C}$, livello dei globuli bianchi elevato). Anche le ABSSSI nei pazienti immunocompromessi sono considerate severe. Le infezioni della cute lievi vengono generalmente risolte con incisione e drenaggio, mentre le ABSSSI moderate potrebbero trarre beneficio dall'incisione e dal drenaggio ma comunemente richiedono un breve ciclo di terapia antibiotica, con conseguente ricovero di breve durata. Nei pazienti con infezioni severe, la rivalutazione clinica viene di solito eseguita 72 ore dopo il ricovero (dimissione il giorno 4 o osservazione prolungata fino al giorno 8), mentre la durata del trattamento è pari a 7-14 giorni (dimissione il giorno 8 o osservazione prolungata, corrispondente a una ospedalizzazione >8 giorni). I pazienti con infezione moderata (nonsevera) sono generalmente ricoverati per $\leq 2-3$ giorni (3-5).

Nonostante la molteplicità dei trattamenti esistenti per la cura delle ABSSSI, la crescente presenza di batteri S. aureus meticillino-resistenti (MRSA) e la loro notevole resistenza alle attuali terapie hanno reso necessario un incremento delle ospedalizzazioni e, dunque, un aumento dei costi.

Ad oggi, le terapie consigliate prevedono, in presenza di ABSSSI non purulenta, I'utilizzo di antibiotici beta-lattamici e clindamicina nei casi di moderata entità e l'utilizzo di vancomicina associata a piperacillina nei casi più gravi; invece per le ABSSSI purulente (che comprendono quelle causate da MRSA) è raccomandato l'utilizzo di doxiciclina o trimetoprim/ sulfametossazolo per i casi di moderata entità e l'utilizzo di vancomicina, daptomicina, linezolid, telavancina o ceftarolina per i casi gravi (4-9).

La dalbavancina è una nuova terapia antibiotica basata su un lipoglicopeptide a lunga durata, approvato dalla FDA nel maggio 2014 e dall'Agenzia Europea per i Medicinali (EMA) nel febbraio 2015 [VER001-9 (10), DISCOVER 1 (NCT01339091) e DISCOVER 2 (NCT01431339 (11)].

Ciò che rende questo farmaco innovativo è il suo effetto battericida e la sua bassa propensione a selezionare resistenze, che gli permettono di contrastare molti patogeni Grampositivi, compresi MRSA, lo $S$. aureus meticillino-sensibile (MSSA) e gli streptococchi.

In particolare, il crescente interesse nei confronti di questa nuova terapia è dovuto ad alcune caratteristiche che la contraddistinguono rispetto agli altri farmaci presenti sul mercato; la dalbavancina infatti è $4 / 8$ volte più potente rispetto alla vancomicina (Standard of Care, SoC) (12) e presenta un'emivita che può durare fino a 14 giorni (13), che consente di poter pianificare nel miglior modo possibile l'ospedalizzazione dei pazienti con ABSSSI. Inoltre, il regime di somministrazione, che inizialmente prevedeva due infusioni (una al giorno $1 \mathrm{e}$ una al giorno 8), è stato sostituito da un'unica soluzione al giorno 1 (14), che garantendo la stessa efficacia permette al paziente di essere dimesso anticipatamente e di completare il trattamento fuori dalla struttura ospedaliera.

Lo scopo di questo studio è stato quello di condurre un'analisi di costo-utilità per confrontare l'utilizzo della dalbavancina per la gestione dei pazienti con ABSSSI non severa rispetto alla terapia antibiotica standard nella prospettiva del Servizio Sanitario Nazionale (SSN).

\section{Metodi}

Il modello è stato sviluppato considerando come misura di outcome gli anni di vita aggiustati per la qualità (Quality Adjusted Life Years, QALYs). II risultato del confronto della dalbavancina con la terapia antibiotica standard in termini di costi ed efficacia è espresso mediante il rapporto incrementale di costo-efficacia (ICER). La prospettiva utilizzata è stata quella del Servizio Sanitario Nazionale (SSN), dunque sono stati considerati solamente i costi sanitari diretti. L'analisi di costo-utilità è stata condotta mediante lo sviluppo di un albero decisionale probabilistico con un follow-up di 30 giorni, in grado di simulare il percorso terapeutico di un paziente trattato rispettivamente con dalbavancina o con SoC $(15,16)$. L'albero decisionale è stato sviluppato a partire da uno studio recentemente pubblicato condotto in tre Paesi europei, tra cui l'Italia, al fine di stimare l'impatto economico associato alla gestione ospedaliera con dalbavancina, rispetto alla terapia antibiotica standard, dei pazienti con ABSSSI non severa (17). II modello ha considerato tre stati di salute mutualmente esclusivi, basati sul numero di notti trascorse in ospedale per il monitoraggio e il trattamento della malattia.

Le probabilità e le utilità associate a ciascun ramo dell'albero decisionale sono state in parte reperite in letteratura e in parte, laddove non presenti, stabilite da clinici esperti. Nell'analisi sono stati considerati i costi dei farmaci e quelli dell'ospedalizzazione per il primo ricovero e per i successivi ricoveri, questi ultimi effettuati conseguentemente a un peggioramento della condizione clinica del paziente.

\section{Albero decisionale}

L'albero decisionale, sviluppato nello studio europeo al fine di simulare il percorso terapeutico dei pazienti con ABSSSI non severa trattati con dalbavancina o con SoC (Figura 1), risultava costituito da tre nodi probabilistici. Al primo nodo probabilistico è stato assegnato un intervallo temporale pari a 5 giorni, al secondo nodo probabilistico un intervallo pari a 1 giorno, mentre al terzo nodo è stato attribuito un intervallo temporale pari a 24 giorni.

Successivamente alla somministrazione in Pronto soccorso di un trattamento antibiotico empirico per le infezioni da Gram-positivi con dalbavancina o con una terapia antibiotica 


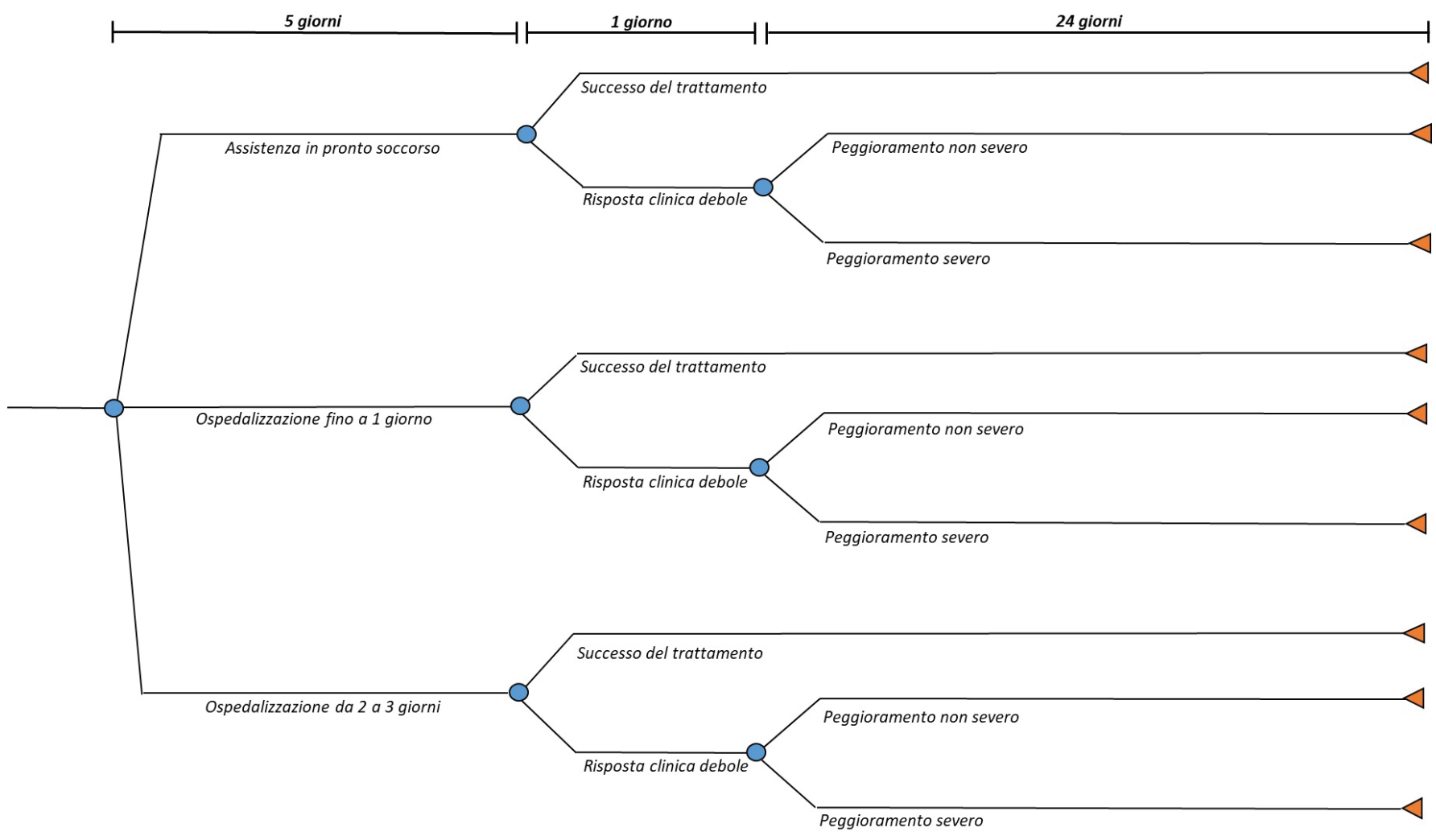

Fig. 1 - Albero decisionale tratto dallo studio europeo (17).

standard, il paziente avrà una certa probabilità di ricadere in uno dei seguenti gruppi (primo nodo probabilistico):

- assistenza in Pronto soccorso;

- ospedalizzazione fino a 1 giorno;

- ospedalizzazione da 2 a 3 giorni.

A partire da ciascuno di questi tre gruppi è stato definito un secondo nodo probabilistico, tramite il quale è stata effettuata un'ulteriore suddivisione di ogni gruppo in:

- pazienti per cui il trattamento ha avuto successo, dunque non incorreranno in un ulteriore ricovero;

- pazienti per cui il trattamento non ha avuto successo e che dunque incorreranno in un ulteriore ricovero.

Infine, per i pazienti per i quali il trattamento non ha avuto successo, è stato definito un terzo nodo probabilistico tale da suddividere i pazienti in:

- pazienti con peggioramento non severo;

- pazienti con peggioramento severo.

\section{Probabilità dell'albero decisionale}

Le probabilità relative alla SoC sono state ottenute dallo studio europeo sopra citato (17) condotto mediante l'analisi del database amministrativo delle schede di dimissione ospedaliera (SDO) per il periodo dal 2006 al 2014 (Tabella I e Tabella A disponibile nei materiali supplementari).
L'introduzione di dalbavancina all'interno del modello consente, grazie alla sua metodologia di somministrazione, una modificazione sia nel setting assistenziale (gestione del paziente in Pronto soccorso) sia una potenziale riduzione dei pazienti che accedono al reparto (Tabella I). Nel nostro modello si assume che il $90 \%$ dei soggetti trattati con dalbavancina ottenga una riduzione delle giornate di degenza. In particolare, per i soggetti che con SoC necessitavano di 0-1 giorno di ospedalizzazione, dalbavancina permette una dimissione direttamente dal Pronto soccorso. Analogamente, per i soggetti che con SoC necessitavano di una ospedalizzazione di 2-3 notti, il trattamento innovativo consente una dimissione direttamente dal Pronto soccorso nel 30\% dei casi, mentre per la restante quota parte riduce la degenza ospedaliera a una sola notte.

\section{Utilità}

Le utilità associate a ciascuno stato di salute dell'albero decisionale sono state definite sulla base di assunzioni in quanto dalla letteratura non sono emersi dati a supporto.

Le utilità associate a ciascuno stato di salute dell'albero decisionale sono state definite a partire dalle utilità assunte per i seguenti stati di salute:

- ai soggetti non malati e di conseguenza non trattati è stata attribuita un'utilità pari a 0,9 (indice di una buona qualità di vita);

- ai pazienti trattati a casa (ossia coloro ai quali viene somministrata una dose del farmaco in Pronto soccorso e 
TABELLA I - Probabilità dell'albero decisionale e stime di efficacia di dalbavancina

\begin{tabular}{|c|c|c|c|c|}
\hline $\begin{array}{l}\text { Pazienti con ABSSSI non severa trattati con SoC } \\
\text { (primo nodo probabilistico) }\end{array}$ & Stima (\%) & $\begin{array}{c}\text { Errore } \\
\text { standard }\end{array}$ & Distribuzione & Fonte \\
\hline Assistenza in Pronto soccorso & 0,00 & & & \multirow{3}{*}{$\begin{array}{l}\text { Stime da database } \\
\text { SDO (18) }\end{array}$} \\
\hline Ospedalizzazione fino a 1 giorno & 41,00 & 0,021 & Beta & \\
\hline Ospedalizzazione da 2 a 3 giorni & 59,00 & 0,030 & Beta & \\
\hline Somministrazione di dalbavancina & Stima (\%) & & & Fonte \\
\hline $\begin{array}{l}\text { Probabilità dei pazienti ospedalizzati 2-3 giorni di essere dimessi } \\
\text { in Pronto soccorso }\end{array}$ & 30,00 & - & - & $\begin{array}{l}\text { Stime da expert } \\
\text { opinion (18) }\end{array}$ \\
\hline $\begin{array}{l}\text { Pazienti con ABSSSI non severa trattati con dalbavancina } \\
\text { (primo nodo probabilistico) }\end{array}$ & Stima (\%) & $\begin{array}{l}\text { Errore } \\
\text { standard }\end{array}$ & Distribuzione & Fonte \\
\hline Assistenza in Pronto soccorso & 52,80 & 0,026 & Beta & Calcolo \\
\hline \multicolumn{5}{|l|}{ Assistenza in Pronto soccorso } \\
\hline Successo del trattamento & 98,50 & - & - & \multirow{2}{*}{$\begin{array}{l}\text { Stime da database } \\
\text { SDO (18) }\end{array}$} \\
\hline Risposta clinica debole & 1,50 & - & - & \\
\hline \multicolumn{5}{|l|}{ Ospedalizzazione fino a 1 giorno } \\
\hline Successo del trattamento & 98,50 & - & - & \multirow{2}{*}{$\begin{array}{l}\text { Stime da database } \\
\text { SDO (18) }\end{array}$} \\
\hline Risposta clinica debole & 1,50 & - & - & \\
\hline \multicolumn{5}{|l|}{ Ospedalizzazione da $\mathbf{2}$ a $\mathbf{3}$ giorni } \\
\hline Successo del trattamento & 99,10 & - & - & $\begin{array}{l}\text { Stime da database } \\
\text { SDO (18) }\end{array}$ \\
\hline \multicolumn{5}{|l|}{ Ospedalizzazione fino a 1 giorno } \\
\hline Peggioramento non severo & 94,80 & 0,047 & Beta & \multirow{2}{*}{$\begin{array}{l}\text { Stime da database } \\
\text { SDO (18) }\end{array}$} \\
\hline Peggioramento severo & 5,20 & 0,003 & Beta & \\
\hline \multicolumn{5}{|l|}{ Ospedalizzazione da 2 a 3 giorni } \\
\hline Peggioramento non severo & 90,50 & 0,045 & Beta & \multirow{2}{*}{$\begin{array}{l}\text { Stime da database } \\
\text { SDO (18) }\end{array}$} \\
\hline Peggioramento severo & 9,50 & 0,005 & Beta & \\
\hline
\end{tabular}

poi trascorrono il resto del percorso terapeutico a casa) è stata attribuita un'utilità pari a 0,8 (leggermente inferiore rispetto a coloro che non contraggono la malattia in quanto si tratta di casi di ABSSSI non severa);

- ai pazienti che a causa della malattia devono essere ospedalizzati (fino a 1 o fino a 3 giorni) è stata attribuita un'utilità pari a 0,5 (in quanto, dovendo trascorrere del tempo in ospedale, non risolvono immediatamente il loro problema e vedono peggiorare la loro qualità di vita);
- ai pazienti per i quali il trattamento ha avuto successo è stata attribuita un'utilità pari a 0,8 , mentre ai pazienti per i quali il trattamento non ha avuto successo è stata attribuita un'utilità pari a 0,6 ;

- infine, tra i pazienti caratterizzati da una risposta debole al trattamento, a quelli che manifestano un peggioramento non severo della condizione di salute è stata attribuita una disutilità di $-0,1$, mentre ai pazienti che manifestano un peggioramento severo della condizione di salute è stata attribuita una disutilità di -0,2 (Tabella II). 
TABELLA II - Utilità

\begin{tabular}{|c|c|c|c|c|}
\hline Stato di salute & Utilità & Errore standard & Distribuzione & Fonte \\
\hline Pazienti non trattati & 0,9 & 0,045 & Beta & \\
\hline Pazienti trattati a casa & 0,8 & 0,040 & Beta & \\
\hline Pazienti trattati con necessità di ospedalizzazione & 0,5 & 0,025 & Beta & \\
\hline Pazienti in cui il trattamento ha avuto successo & 0,8 & 0,040 & Beta & Assunzione \\
\hline Pazienti con risposta clinica debole & 0,6 & 0,030 & Beta & \\
\hline Pazienti con peggioramento non severo della condizione clinica & $-0,1$ & $-0,005$ & Beta & \\
\hline Pazienti con peggioramento severo della condizione clinica & $-0,2$ & $-0,010$ & Beta & \\
\hline
\end{tabular}

TABELLA III - Utilità associate agli stati di salute presenti nell'albero decisionale

\begin{tabular}{lc}
\hline Stato di salute & Utilità ponderata \\
\hline Assistenza in Pronto soccorso & 0,880 \\
Assistenza in Pronto soccorso con peggioramento non severo della condizione clinica & 0,888 \\
Assistenza in Pronto soccorso con peggioramento severo della condizione clinica & 0,871 \\
Ospedalizzazione fino a 1 giorno & 0,800 \\
Ospedalizzazione fino a 1 giorno con peggioramento non severo della condizione clinica & 0,871 \\
Ospedalizzazione fino a 1 giorno con peggioramento severo della condizione clinica & 0,854 \\
Ospedalizzazione da 2 a 3 giorni & 0,640 \\
Ospedalizzazione da 2 a 3 giorni con peggioramento non severo della condizione clinica & 0,838 \\
Ospedalizzazione da 2 a 3 giorni con peggioramento severo della condizione clinica & 0,821 \\
Successo del trattamento & 0,800 \\
Risposta clinica debole & 0,600 \\
\hline
\end{tabular}

TABELLA IV - Parametri di costo (SoC) tratti dallo studio europeo (17)

\begin{tabular}{|c|c|c|c|}
\hline Stato del modello & $\begin{array}{c}\text { Costo per } \\
\text { paziente }(€)\end{array}$ & $\begin{array}{c}\text { Errore } \\
\text { standard }\end{array}$ & Distribuzione \\
\hline Assistenza in Pronto soccorso & 0,0 & - & - \\
\hline Assistenza in Pronto soccorso con peggioramento severo della condizione clinica & $3.163,0$ & 158,2 & Gamma \\
\hline Ospedalizzazione fino a 1 giorno & 490,0 & 24,5 & Gamma \\
\hline Ospedalizzazione fino a 1 giorno con peggioramento severo della condizione clinica & $3.163,0$ & 158,2 & Gamma \\
\hline Ospedalizzazione da 2 a 3 giorni & $2.735,3$ & 136,8 & Gamma \\
\hline Ospedalizzazione da 2 a 3 giorni con peggioramento non severo della condizione clinica & $2.226,1$ & 111,3 & Gamma \\
\hline Ospedalizzazione da 2 a 3 giorni con peggioramento severo della condizione clinica & $2.748,7$ & 137,4 & Gamma \\
\hline
\end{tabular}

SoC $=$ Standard of Care

Le utilità relative a ciascun ramo dell'albero decisionale, riportate nella Tabella III, sono state ottenute pesando le utilità definite in precedenza (Tabella II) per i giorni che il paziente trascorre in ciascuna fase appartenente allo stato di salute (Tabella $B$ disponibile nel materiale supplementare).

\section{Parametri di costo}

I costi relativi ai ricoveri (Tabella IV) sono stati ottenuti dallo studio di impatto sul budget europeo (17). In particolare, sono stati stimati i costi di ri-ospedalizzazione considerando una durata della degenza $<4$ giorni per i pazienti con 
peggioramento clinico non severo e una durata della degenza $\geq 4$ giorni per i pazienti con un peggioramento clinico severo. Come assunto anche nello studio europeo, il costo relativo al trattamento con SoC è stato considerato pari a zero poiché tale terapia presenta costi relativamente bassi e già contenuti nella tariffa dei DRG associati ai codici ICD-9 considerati. Il costo di dalbavancina è risultato pari a $€ 1.161$ per l'intera somministrazione (18) (Tabella IV).

\section{Analisi di sensibilitò}

Al fine di considerare la variabilità intrinseca dei dati utilizzati nel modello, sono state condotte un'analisi di sensibilità deterministica a una via (one-way Deterministic Sensitivity Analysis, DSA) e un'analisi di sensibilità probabilistica (Probabilistic Sensitivity Analysis, PSA). La prima analisi consiste nel far variare un parametro di input alla volta, in funzione della variabilità riscontrata in letteratura o assunta dagli autori, al fine di valutare l'impatto di ciascun parametro sui risultati dell'analisi. In questo caso per ciascun parametro si è assunta una variazione del $5 \%$. Nella seconda analisi tutti i parametri di input vengono fatti variare contemporaneamente (assumendo in questo caso una variazione del $5 \%$ ), ciascuno secondo una determinata distribuzione di probabilità (Gamma per i parametri di costo, Beta per le probabilità e le utilità). Sono state condotte 1.000 simulazioni Montecarlo al fine di generare la curva di accettabilità di costo-efficacia (Cost Effectiveness Acceptability Curve, CEAC) per la dalbavancina. Tale curva esprime la probabilità di costo-efficacia della dalbavancina (rispetto alla SoC) in base alla disponibilità a pagare del SSN; un valore di ICER al di sotto di $€ 30.000$ per QALY guadagnato è stato assunto come valore soglia di costo-efficacia.

Infine, sono state effettuate delle simulazioni sul parametro di efficacia di dalbavancina al fine di valutare il break-even point (BPT). II BPT è stato definito come il livello di efficacia minimo di dalbavancina per cui il trattamento possa ritenersi costo-efficace rispetto alla SoC (considerando una soglia di disponibilità a pagare pari $a € 30.000$ per QALY guadagnato).

\section{Risultati}

Dall'analisi è emerso come l'utilizzo di dalbavancina in pazienti con ABSSSI non severa rispetto al trattamento con SoC possa tradursi in una riduzione dei costi $(-€ 291,6$ per paziente trattato) e in un incremento di QALYs $(+0,0018$ per paziente trattato) (Tabella V).

Dall'osservazione del piano di costo-efficacia (Figura 2), che riporta le 1.000 simulazioni Montecarlo effettuate, è possibile notare come in circa il $99,7 \%$ delle simulazioni effettuate la dalbavancina sia risultata dominante rispetto alla SoC.

TABELLA V - Risultati di costo-utilità

\begin{tabular}{lccccc}
\hline & Costi totali $(€)$ & QALYs totali & Costi incrementali $(€)$ & QALYs incrementali & ICER \\
\hline SoC & $1.834,7$ & 0,0645 & - & - & - \\
Dalbavancina & $1.543,0$ & 0,0663 & $-291,6$ & 0,0018 & Dominante \\
\hline
\end{tabular}

ICER = Incremental Cost-Effectiveness Ratio; QALYs = Quality-Adjusted Life Years; SoC = Standard of Care

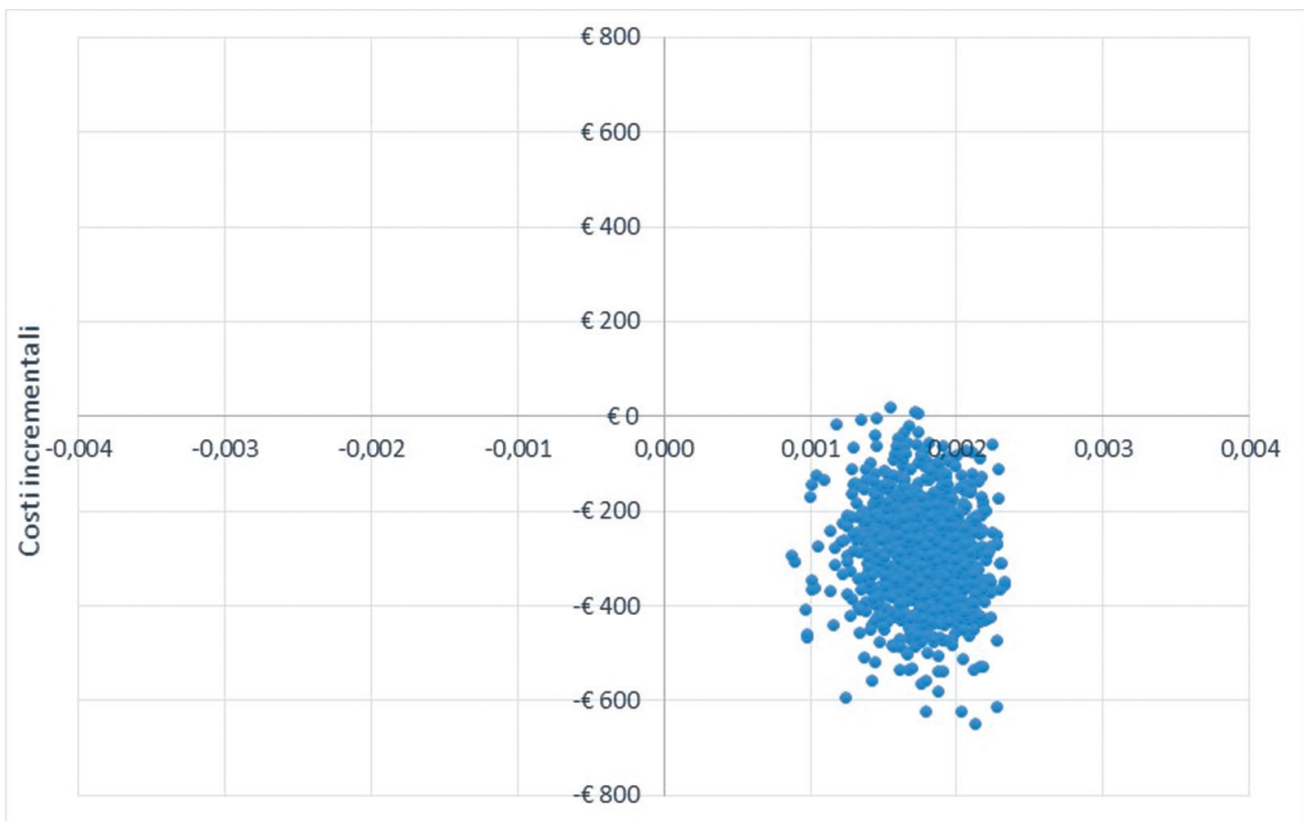

Fig. 2 - Piano di costo-efficacia.

QALYs incrementali 


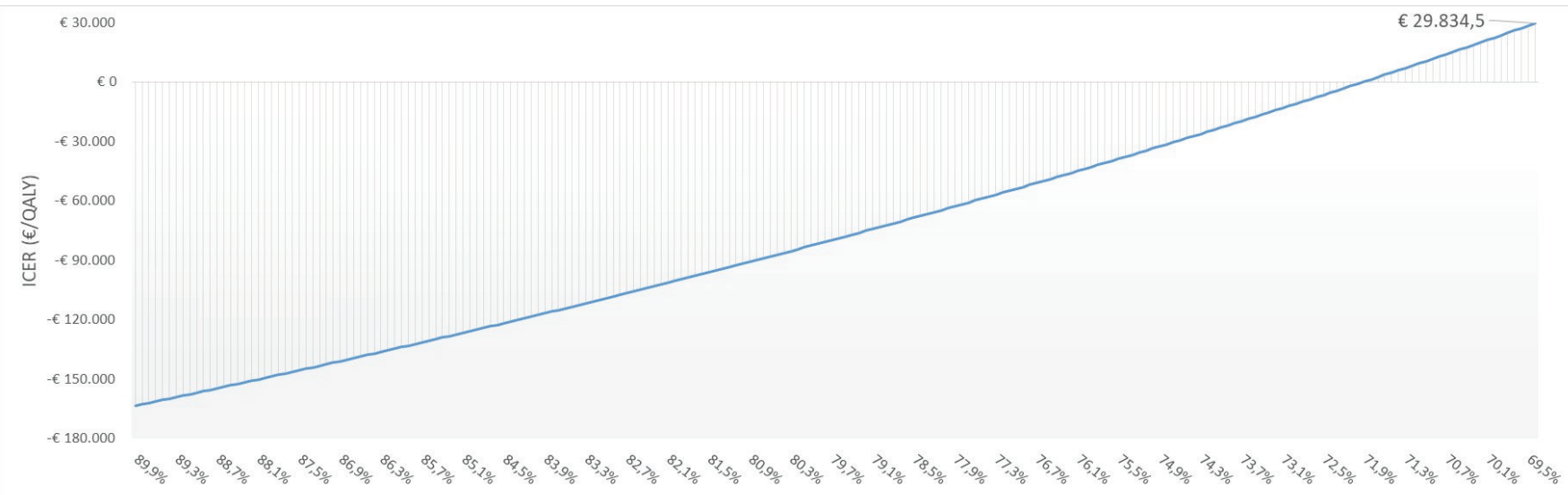

EFFICACIA DALBAVANCINA - CASO-BASE $=90 \%$

Fig. 3 - Analisi di break-even point.

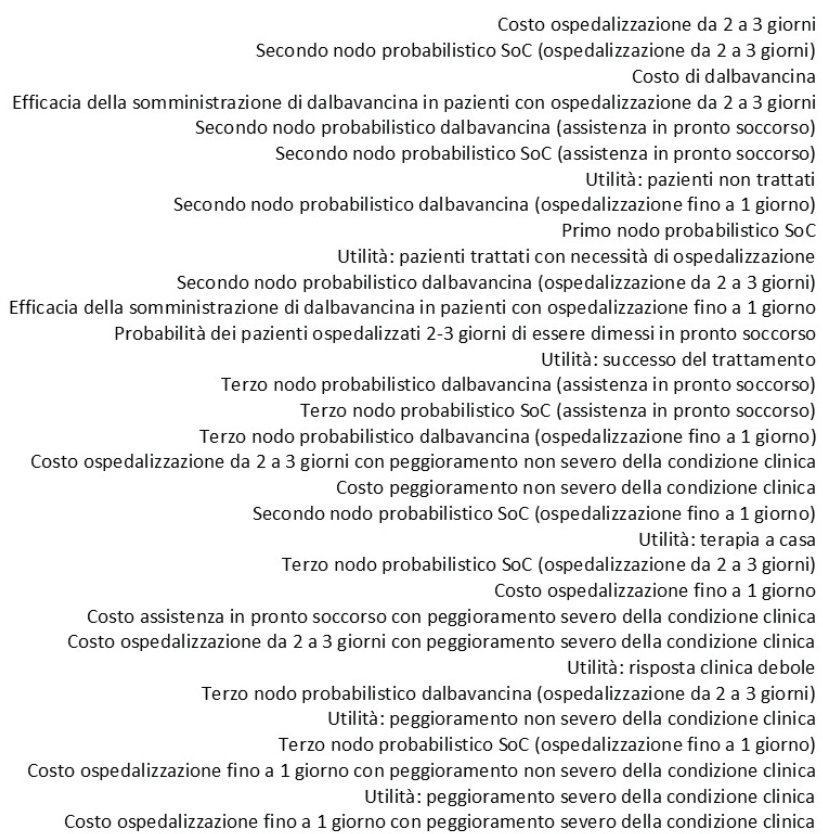

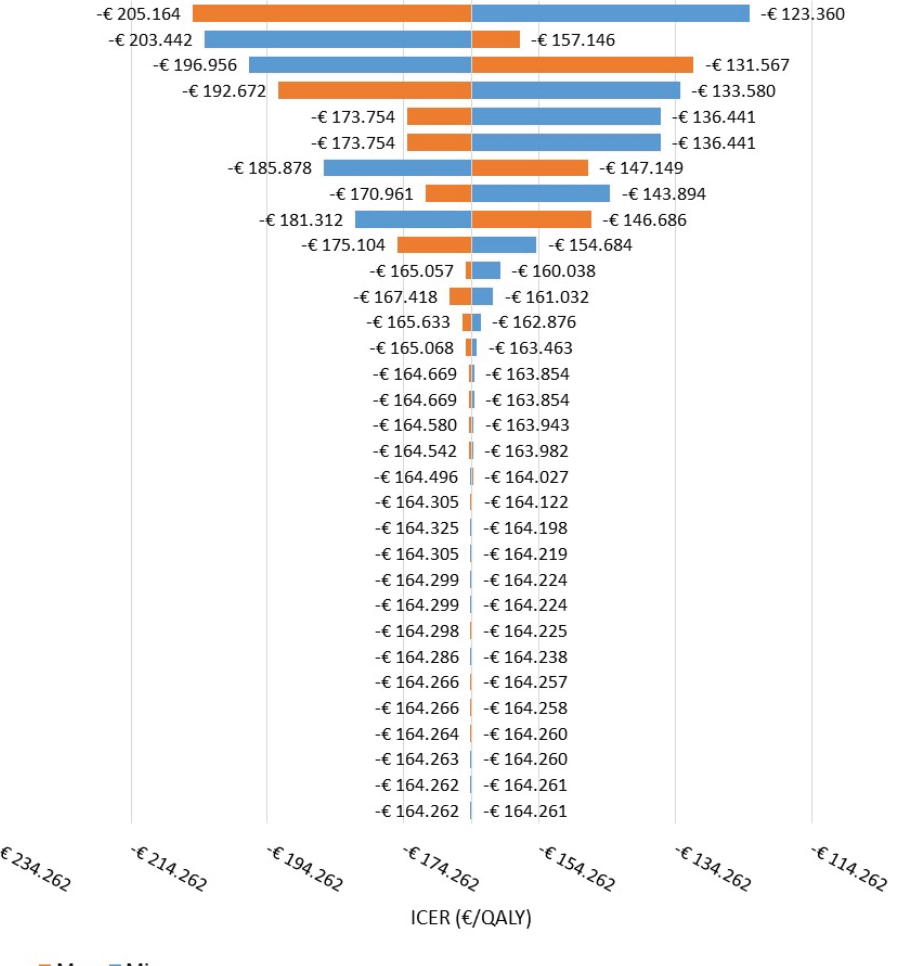

$\square \operatorname{Max}=\operatorname{Min}$

Fig. 4 - Grafico tornado.

Le simulazioni effettuate sul parametro di efficacia di dalbavancina (caso-base $=90 \%$, sia per i pazienti ospedalizzati 0-1 giorno, sia per i pazienti ospedalizzati 2-3 giorni) hanno fatto emergere come la dalbavancina possa risultare un trattamento costo-efficace (valore di ICER inferiore ad una soglia di disponibilità a pagare pari a $€ 30.000$ per QALY guadagnato) rispetto alla SoC in corrispondenza di valori di efficacia maggiori del 69,4\% (Figura 3).

Il risultato dell'analisi di sensibilità deterministica a una via è rappresentato nel grafico tornado (Figura 4): il parametro cui è risultato associato il maggiore impatto sui risultati è quello relativo al costo dell'ospedalizzazione di 2-3 notti, seguito dalle probabilità relative al secondo nodo probabilistico per l'ospedalizzazione di 2-3 notti e dal costo di dalbavancina.

\section{Discussione}

Il trattamento di pazienti affetti da ABSSSI richiede tempi di ospedalizzazione molto lunghi che, oltre ad apportare un peggioramento alla qualità di vita del paziente stesso, comportano costi elevati per il SSN. 
Uno studio retrospettivo, condotto negli Stati Uniti su pazienti con diagnosi di ABSSSI da $S$. aureus, ha stimato una degenza media per paziente di circa 6 giorni (19).

In Europa è stato osservato come, in caso di infezione complicata, la degenza media sia pari a 18,5 giorni $(27,7$ giorni in caso di infezione da MRSA) (20). Si tratta dunque di una patologia che nella sua modalità di gestione ospedaliera comporta un peso notevole sui budget dei Servizi sanitari.

A livello nazionale, lo studio di Marcellusi et al. del 2020 condotto in tre Paesi europei mediante l'impiego dei database amministrativi ha stimato una spesa annua totale per la gestione dei pazienti con ABSSSI in Italia (circa 5.396 soggetti) pari a circa $€ 9,9$ milioni (17). L'analisi di impatto sul budget ha permesso di stimare come l'introduzione di dalbavancina per il trattamento di pazienti con ABSSSI non severa in Italia possa comportare una riduzione sia del numero di pazienti ospedalizzati, sia della durata della degenza ospedaliera (-230 giorni e -677 giorni per 1.000 pazienti ospedalizzati al primo anno e al terzo anno dall'introduzione di dalbavancina sul mercato), generando una riduzione della spesa cumulata a carico del SSN al terzo anno dalla sua introduzione pari a circa $€ 2,1$ milioni.

Alla luce di questi dati, si evidenzia la necessità di trattamenti ugualmente efficaci a quelli già presenti sul mercato ma che permettano al contempo di migliorare la gestione del paziente. L'analisi ha mostrato come la dalbavancina, prevedendo un'unica somministrazione e riducendo, così come ipotizzato da esperti clinici, il numero di pazienti ospedalizzati da 0 a 1 giorno e da 2 a 3 giorni, possa risultare una strategia di trattamento meno costosa e più efficace (dominante) rispetto allo SoC per il trattamento dei pazienti con ABSSSI non severa. Tali risultati rappresentano il frutto dell'elaborazione di stime ottenute dalla letteratura e dall'opinione di clinici esperti, soprattutto con riferimento all'efficacia di dalbavancina; infatti, a nostra conoscenza, non risultano ancora presenti in letteratura studi osservazionali che abbiano valutato l'efficacia di dalbavancina in questo specifico setting di pazienti. Tale aspetto rappresenta sicuramente un primo limite dello studio; le simulazioni effettuate sul parametro di efficacia di dalbavancina hanno dimostrato come il livello minimo di efficacia affinché la dalbavancina possa risultare una strategia terapeutica costo-efficace rispetto allo SoC sia pari al 69,4\% (considerando una soglia di disponibilità a pagare pari a $€ 30.000$ per QALY guadagnato). Un ulteriore limite dello studio risulta caratterizzato dalle ipotesi effettuate per la stima delle utilità relative a ciascuno stato di salute appartenente all'albero decisionale, in quanto dalla letteratura non sono emersi studi condotti sulla qualità di vita per questo specifico setting di pazienti. Per far fronte a tutti gli assunti effettuati all'interno del modello, è stata condotta un'analisi di sensibilità a una via proprio per mostrare i parametri che maggiormente vanno a impattare sui risultati e $i$ valori di ICER che si potrebbero ottenere considerando una riduzione o un aumento di ciascun parametro pari al $5 \%$.

\section{Conclusioni}

Le ABSSSI rappresentano un importante problema sanitario con considerevoli ricadute economiche. L'analisi ha mostrato come il trattamento con dalbavancina possa rappresentare una strategia costo-efficace rispetto alle terapie antibiotiche standard in pazienti con ABSSSI non severa. Sono necessari studi osservazionali da condurre in questo specifico setting di pazienti, volti a valutare l'efficacia di dalbavancina dal punto di vista della dimissione dal Pronto soccorso e della riduzione del numero di giornate di degenza.

\section{Disclosures}

Conflict of interest: The authors declare they have no conflict of interest related to the article.

Financial support: This study was supported by an unconditional grant from Angelini SpA.

\section{Bibliografia}

1. The United States Food and Drug Administration. Guidance for Industry Acute Bacterial Skin and Skin Structure Infections: Developing Drugs for Treatment. 2013. Online

2. Stevens DL, Bisno AL, Chambers HF et al; Infectious Diseases Society of America. Practice guidelines for the diagnosis and management of skin and soft tissue infections: 2014 update by the Infectious Diseases Society of America. Clin Infect Dis. 2014;59(2):e10-e52. CrossRef Medline

3. Garau J, Ostermann H, Medina J et al; REACH study group. Current management of patients hospitalized with complicated skin and soft tissue infections across Europe (2010-2011): assessment of clinical practice patterns and real-life effectiveness of antibiotics from the REACH study. Clin Microbiol Infect. 2013;19(9):E377-E385. CrossRef Medline

4. Pollack CV Jr, Amin A, Ford WT Jr et al. Acute bacterial skin and skin structure infections (ABSSSI): practice guidelines for management and care transitions in the emergency department and hospital. J Emerg Med. 2015;48(4):508-19. CrossRef Medline

5. Esposito S, Bassetti M, Concia E et al; Italian Society of Infectious and Tropical Diseases. Diagnosis and management of skin and soft-tissue infections (SSTI). A literature review and consensus statement: an update. J Chemother. 2017;29(4):197-214. CrossRef Medline

6. Russo A, Concia E, Cristini $F$ et al. Current and future trends in antibiotic therapy of acute bacterial skin and skin-structure infections. Clin Microbiol Infect. 2016;22(suppl 2):S27-S36. CrossRef Medline

7. Hall RG II, Smith WJ, Putnam WC, Pass SE. An evaluation of tedizolid for the treatment of MRSA infections. Expert Opin Pharmacother. 2018;19(13):1489-94. CrossRef Medline

8. Bassetti M, Peghin M, Castaldo N, Giacobbe DR. The safety of treatment options for acute bacterial skin and skin structure infections. Expert Opin Drug Saf. 2019;18(8):635-50. CrossRef Medline

9. Guervil DJ, Kaye KS, Hassoun A et al. Ceftaroline fosamil as first-line versus second-line treatment for acute bacterial skin and skin structure infections (ABSSSI) or community-acquired bacterial pneumonia (CABP). J Chemother. 2016;28(3):180-6. CrossRef Medline

10. Jauregui LE, Babazadeh S, Seltzer E et al. Randomized, doubleblind comparison of once-weekly dalbavancin versus twicedaily linezolid therapy for the treatment of complicated skin and skin structure infections. Clin Infect Dis. 2005;41(10):1407-15. CrossRef Medline

11. Boucher HW, Wilcox M, Talbot GH et al. Once-weekly dalbavancin versus daily conventional therapy for skin infection. $\mathrm{N}$ Engl J Med. 2014;370(23):2169-79. CrossRef Medline 
12. McCurdy SP, Jones RN, Mendes RE et al. In vitro activity of dalbavancin against drug-resistant Staphylococcus aureus isolates from a global surveillance program. Antimicrob Agents Chemother. 2015;59(8):5007-9. CrossRef Medline

13. Bassetti M, Peghin M, Carnelutti A, Righi E. The role of dalbavancin in skin and soft tissue infections. Curr Opin Infect Dis. 2018;31(2):141-7. CrossRef Medline

14. European Medicines Agency (EMA). Xydalba. Riassunto delle caratteristiche del prodotto. Online

15. Guest JF, Esteban J, Manganelli AG et al. Comparative efficacy and safety of antibiotics used to treat acute bacterial skin and skin structure infections: results of a network meta-analysis. PLoS One. 2017;12(11):e0187792. CrossRef Medline

16. Gonzalez PL, Rappo U, Akinapelli K et al. Treatment of acute bacterial skin and skin structure infection with single-dose dalbavancin in persons who inject drugs. Drugs Context. 2018;7:212559. CrossRef Medline
17. Marcellusi A, Bini C, Andreoni M et al. Budget impact analysis of dalbavancin in the treatment of acute bacterial skin and skin structure infections in three European countries. Clin Drug Investig. 2020;40(4):305-18. CrossRef Medline

18. Marcellusi A, Viti R, Sciattella P et al. Economic evaluation of the treatment of Acute Bacterial Skin and Skin Structure Infections (ABSSSIs) from the national payer perspective: introduction of a new treatment to the patient journey. A simulation of three European countries. Expert Rev Pharmacoecon Outcomes Res. 2019;19(5):581-99. CrossRef Medline

19. Seaton RA, Johal S, Coia JE et al. Economic evaluation of treatment for MRSA complicated skin and soft tissue infections in Glasgow hospitals. Eur J Clin Microbiol Infect Dis. 2014;33:305-11. CrossRef Medline

20. Itani KM, Merchant S, Lin SJ et al. Outcomes and management costs in patients hospitalized for skin and skin-structure infections. Am J Infect Control. 2011;39(1):42-9. CrossRef Medline 